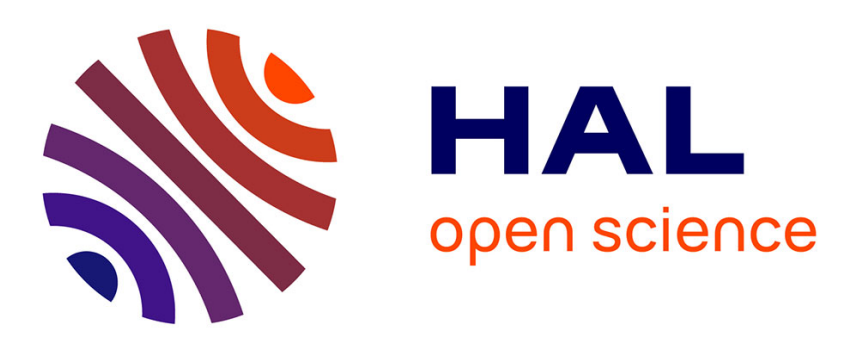

\title{
M-theta Method on Mode I and II Failure of Two Cameroonian Hardwoods in Bending
} Hervice Kouefouet, Jeanne Sandrine Takam Mabekou, Joseph Albert Fosting Mukam, Pierre Kisito Talla, Rostand Moutou Pitti

\section{- To cite this version:}

Hervice Kouefouet, Jeanne Sandrine Takam Mabekou, Joseph Albert Fosting Mukam, Pierre Kisito Talla, Rostand Moutou Pitti. M-theta Method on Mode I and II Failure of Two Cameroonian Hardwoods in Bending. European Journal of Engineering Research and Science, 2019, 4 (3), pp.176-182. 10.24018/ejers.2019.4.3.1175 . hal-02116813

\section{HAL Id: hal-02116813 https://hal.science/hal-02116813}

Submitted on 1 May 2019

HAL is a multi-disciplinary open access archive for the deposit and dissemination of scientific research documents, whether they are published or not. The documents may come from teaching and research institutions in France or abroad, or from public or private research centers.
L'archive ouverte pluridisciplinaire HAL, est destinée au dépôt et à la diffusion de documents scientifiques de niveau recherche, publiés ou non, émanant des établissements d'enseignement et de recherche français ou étrangers, des laboratoires publics ou privés. 


\title{
M-THETA METHOD ON MODE I AND II FAILURE OF TWO CAMEROONIAN HARDWOODS IN BENDING
}

Hervice Kouefouet ${ }^{1}$, Jeanne Sandrine Takam Mabekou ${ }^{1}$, Joseph Albert Mukam Fotsing ${ }^{2}$, Pierre Kisito Talla ${ }^{1(*)}$, Rostand Moutou Pitti ${ }^{3}$

${ }^{1}$ UR_2MSP, University of Dschang, Cameroon

${ }^{2}$ ENS, University of Yaoundé I, Cameroon

${ }^{3}$ Université Clermont Auvergne, CNRS, SIGMA Clermont, Institut Pascal, F-63000

Clermont-Ferrand, France

*Author to whom correspondence should be addressed. E-mail: tpierrekisito@yahoo.com

\begin{abstract}
This work deals with the numerical simulation on bending test to characterize two Cameroonian hardwoods under mode I and II loading for different crack lengths. The finite element analysis for fracture in orthotropic medium is developed. The algorithm of fracture is introduced in a finite element software Cast3M. According to the Mtheta method, the calculation of the stress intensity factors and the energy release rate for pure mode I and II fracture are deduced using a SENB (Single Edge Notch Bending) specimen. The path independence of $M \theta$-method on the specimen is confirmed.
\end{abstract}

Keywords: Mode I and II failure; Mtheta method; Finite element analysis; SENB specimen

\section{INTRODUCTION}

The wood species lovoa trichilioides and triplochiton scleroxylon are subject of an increasingly high exploitation and exportation each year in Cameroon and belong to the first five types of hardwoods exploited in the country and used in the various ways [1], [2]. They are very little used for construction, but important for socioeconomic function of the country. Often used in an empirical way, Cameroonian woods are the source of many security and dimension problems, for instance when they are used for the construction of bridges and heavy roof frames. In this context, wood material has a special interest to revive construction sector. Furthermore, due to the economic cost, the design of structure is required not for oversized dimension, but to respect the limit range of the security. Therefore, fracture behavior of wood is important to be more investigated to better understand the crack process in the structure [3].

In this regard, many work have been done to evaluate the characteristics of fracture and their consequences on wood structure facing crack problems. Reference [4] investigated 
in 2002 the influence of the physical and mechanical characteristics on the behavior of wood during machining. The determination of fracture energy and fracture process zone length in Mode I fracture of European spruce wood were reported by [5]. Reference [6] work on fracture energy of wood and root burl wood of Thuya. The mode I fracture of tropical woods using grid method has been developed by [7]. The Influence of loading rate on the mode II fracture toughness of vinyl ester GRP where investigated by [8] in 2017. In this work bending simulation used the Mtheta method introduced by [9]- [11] in the plane constrain for linear elastic fracture mechanics to model crack growth on two Cameroonian hardwoods.

II. LOCAL MECHANICAL FIELDS IN ORTHOTROPIC MEDIUM FOR PLANE CRACK

\section{II.1. FIELDS OF STRESSES AND DISPLACEMENTS}

We considered a thin solid with a constant width subjected to the applied forces in his contour as shown in Fig. 1. The stresses in the $\mathrm{z}$ direction is neglected and are too small in front of $\sigma_{x x}, \sigma_{y y}$ and $\sigma_{x y}$ for continuity reason.

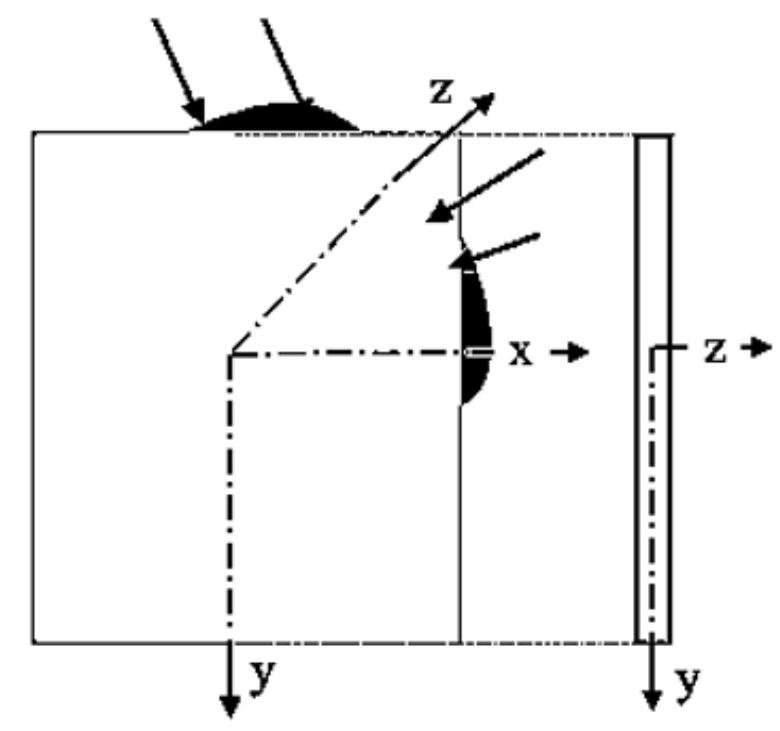

Fig. 1. Solid in a plane stress.

In the absence of the body forces, the equilibrium equations are considering in the form of (1): 


$$
\left\{\begin{array}{l}
\frac{\partial \sigma_{x x}}{\partial x}+\frac{\partial \sigma_{x y}}{\partial y}=0 \\
\frac{\partial \sigma_{x y}}{\partial x}+\frac{\partial \sigma_{y y}}{\partial y}=0
\end{array}\right.
$$

$$
\left\{\begin{array}{l}
\sigma_{x}=\sigma_{x x}=\frac{\partial^{2} \Psi}{\partial y^{2}} \\
\sigma_{y}=\sigma_{y y}=\frac{\partial^{2} \Psi}{\partial x^{2}} \\
\sigma_{x y}=-\frac{\partial^{2} \Psi}{\partial x \partial y}
\end{array}\right.
$$

By introducing the Airy's stress function $\Psi(x, y)$ and taking into account (2) the compatibility equation give (3).

$$
\frac{\partial^{4} \Psi}{\partial x^{4}}+2 \frac{\partial^{4} \Psi}{\partial x^{2} \partial y^{2}}+\frac{\partial^{4} \Psi}{\partial y^{4}}=0
$$

Hence we said $\Psi(x, y)$ satisfies the biharmonic equation (3) for isotropic medium. For orthotropic case let consider the orthotropic principal planes and (4) give the relation between stress and strain.

$$
\left[\begin{array}{l}
\varepsilon_{x x} \\
\varepsilon_{y y} \\
\varepsilon_{x y}
\end{array}\right]=\left(\begin{array}{ccc}
S_{11} & S_{12} & 0 \\
S_{12} & S_{22} & 0 \\
0 & 0 & S_{33}
\end{array}\right) \cdot\left[\begin{array}{c}
\sigma_{x x} \\
\sigma_{y y} \\
\sigma_{x y}
\end{array}\right]
$$

The compatibility equation become with Airy's stress function the equation (5) in plane coordinate.

$$
\frac{\partial^{4} \Psi}{\partial x^{4}} \cdot S_{22}+\frac{\partial^{4} \Psi}{\partial x^{2} \partial y^{2}} \cdot\left(2 S_{12}+S_{33}\right)+\frac{\partial^{4} \Psi}{\partial y^{4}} \cdot S_{11}=0
$$


By setting a change of variables [12], [13] as follow below,

$$
\begin{aligned}
& Z_{1}=x+\alpha_{1} y ; Z_{2}=x+\alpha_{2} y ; \\
& \frac{\partial Z_{i}}{\partial x}=1 ; \frac{\partial Z_{i}}{\partial y}=\alpha_{i}, i \in[1 ; 2] \\
& \Psi=2 . R e\left[\theta_{1}\left(Z_{1}\right)+\psi_{2}\left(Z_{2}\right)\right]
\end{aligned}
$$

The combination of equation (5), (6) and (7) help to introduced (8) as giving below.

$$
2 . \Re e\left[\left(S_{11} \cdot \alpha_{i}^{4}+\left(2 \cdot S_{12}+S_{33}\right) \cdot \alpha_{i}^{2}+S_{22}\right) \cdot \psi_{i}\left(Z_{i}\right)\right]=0
$$

Equation (8) is the characteristic equation deriving from strain integrality condition. Solving (8) give's four solutions in $\alpha_{1} ; \alpha_{2}$ and there complex conjugates $\bar{\alpha}_{1}$ and $\overline{\alpha_{2}}$ following the relation below (9).

$$
\begin{aligned}
& \alpha_{1}=i \sqrt{A} \\
& \alpha_{2}=i \sqrt{B}
\end{aligned}
$$

Where $\mathrm{A}$ and $\mathrm{B}$ are defined as giving by (9a) and (9b):

$$
\begin{aligned}
& A=\frac{1}{2 S_{11}}\left[2 . S_{12}+S_{33}-\sqrt{\left(2 . S_{12}+S_{33}\right)^{2}-4 . S_{11} S_{22}}\right] \\
& B=\frac{1}{2 S_{11}}\left[2 . S_{12}+S_{33}+\sqrt{\left(2 . S_{12}+S_{33}\right)^{2}-4 . S_{11} S_{22}}\right]
\end{aligned}
$$

The elements $S_{11}, S_{12}, S_{22}, S_{33}$ are the elastic compliance tensor components for an orthotropic symmetry. Considering the orthotropic directions (L, R, T), the elastic compliance tensor components are directly related to the elastic orthotropic characteristics for an opening mode solicitation in $(\mathrm{R}, \mathrm{L})$ plane with the crack oriented in the $\mathrm{L}$ direction of fiber. In the plane stress their expression is stated in the form 
$S_{11}=\frac{1}{E_{x}} ; \quad S_{22}=\frac{1}{E_{y}} ; \quad S_{33}=\frac{1}{\mu_{x y}} ; \quad S_{12}=-\frac{v_{x y}}{E_{x}}$

$E_{x}, E_{y}$ are Young modules in $\mathrm{x}$ and $\mathrm{y}$ directions and $\mu_{x y}, v_{x y}$ are respectively the shear module and Poisson coefficient in the considering direction $\mathrm{x}$ and $\mathrm{y}$. From (5) and considering the equilibrium equations, the field of stresses is stated as:

$$
\left\{\begin{array}{l}
\sigma_{x}=2 . R e\left[\alpha_{1}^{2} \phi^{\prime}\left(z_{1}\right)+\alpha_{2}^{2} \chi^{\prime}\left(z_{2}\right)\right] \\
\sigma_{y}=2 \Re R e\left[\phi^{\prime}\left(z_{1}\right)+\chi^{\prime}\left(z_{2}\right)\right] \\
\sigma_{x y}=2 . R e\left[\alpha_{1} \phi^{\prime}\left(z_{1}\right)+\alpha_{2} \chi^{\prime}\left(z_{2}\right)\right]
\end{array}\right.
$$

Where $\phi$ ' and $\chi$ ' denote the derivative.

By combining (10) and (4), we obtain the deformation and by integration we deduced the displacement as follow:

$$
\begin{aligned}
& \left\{\begin{array}{l}
u_{1}=2 . \Re e\left[p_{1} \chi\left(z_{1}\right)+p_{2} \phi\left(z_{2}\right)\right] \\
u_{2}=2 . \Re e\left[q_{1} \chi\left(z_{1}\right)+q_{2} \phi\left(z_{2}\right)\right]
\end{array}\right. \\
& \text { with } p_{i}=S_{11} \alpha_{i}^{2}+S_{12} \text { and } q_{i}=\frac{S_{22}}{\alpha_{i}}+S_{12} \alpha_{i}, \quad i=\{1 ; 2\}
\end{aligned}
$$

\section{II.2. RELATION BETWEEN STRESS INTENSITY FACTOR,} DISPLACEMENTS AND STRESSES

From Airy's function, let set (12) in order to deduce the relation between the stress intensity factor and stress. 
$\left\{\begin{array}{l}\phi(Z)=\sum_{n=1}^{N} A_{n} Z^{\frac{n}{2}} \\ \chi(Z)=\sum_{n=1}^{N} B_{n} Z^{\frac{n}{2}}\end{array}\right.$

With $Z=x+i y=r e^{i \theta}$

$\left\{\begin{array}{l}A_{n}=a^{1}{ }_{n}+i a^{2}{ }_{n} \\ B_{n}=b_{n}^{1}+i b^{2}{ }_{n}\end{array}\right.$

Fig. 2. Crack orientation.

Taking the surface area of the crack free in tension (Fig. 2), for $\Theta= \pm \pi$ permit to deduce that

$$
\sigma_{y}=\sigma_{x y}=0
$$

Therefore (10) and (13) imply that,

$\sigma_{y}+i \sigma_{x y}=\left(1+\beta_{1}\right) \chi^{\prime}\left(z_{1}\right)+\left(1+\beta_{2}\right) \phi^{\prime}\left(z_{2}\right)+\left(1-\beta_{1}\right) \overline{\chi^{\prime}}\left(z_{1}\right)+\left(1-\beta_{2}\right) \overline{\phi^{\prime}}\left(z_{2}\right)$

with $\alpha_{j}=i \beta_{j}, j \in\{1 ; 2\}, Z_{j}=x e^{ \pm i \pi}$ for $\theta= \pm \pi$

For each values of $\Theta,(13)$ and (14) can be presented as follow:

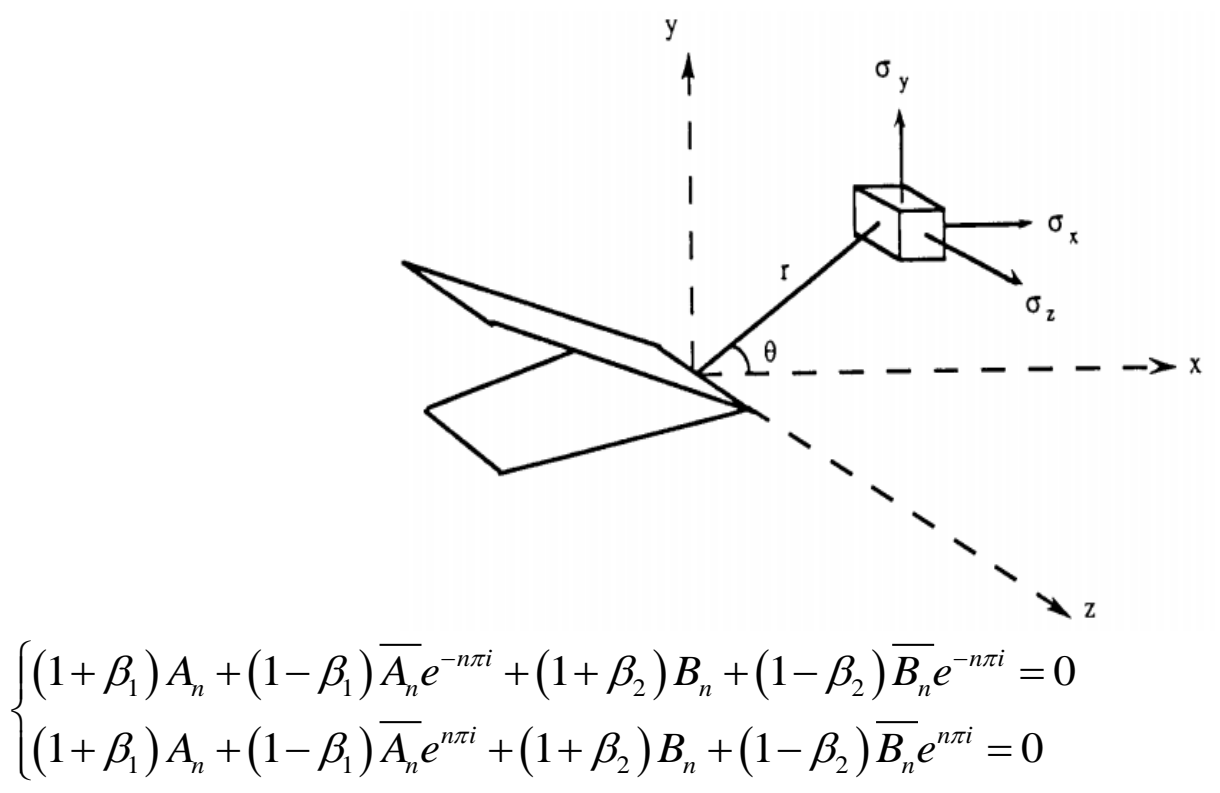

From (15) the relation between $A_{n}$ and $B_{n}$ more over between 
$a_{n}^{1}, b_{n}^{1}$ and $a_{n}^{2}, b_{n}^{2}$

Is obtained by imposing that deformation energy should be finite at the vicinity of the crack, therefore $n=1$ [12], [13]. By comparing to the isotropic medium, the stress intensity factor is related to the field of stresses by (16).

$$
\begin{aligned}
& \sigma_{x}=\frac{K_{I}(\sigma)}{\sqrt{2 \pi r}} \mathfrak{R} e\left[\frac{\alpha_{1} \cdot \alpha_{2}}{\alpha_{1}-\alpha_{2}}\left(\frac{\alpha_{2}}{\sqrt{\rho_{2}}}-\frac{\alpha_{1}}{\sqrt{\rho_{1}}}\right)\right]+\frac{K_{I I}(\sigma)}{\sqrt{2 \pi r}} \mathfrak{R} e\left[\frac{1}{\alpha_{1}-\alpha_{2}}\left(\frac{\alpha_{2}^{2}}{\sqrt{\rho_{2}}}-\frac{\alpha_{1}^{2}}{\sqrt{\rho_{1}}}\right)\right] \\
& \sigma_{y}=\frac{K_{I}(\sigma)}{\sqrt{2 \pi r}} \mathfrak{R} e\left[\frac{1}{\alpha_{1}-\alpha_{2}}\left(\frac{\alpha_{1}}{\sqrt{\rho_{2}}}-\frac{\alpha_{2}}{\sqrt{\rho_{1}}}\right)\right]+\frac{K_{I I}(\sigma)}{\sqrt{2 \pi r}} \mathfrak{R} e\left[\frac{1}{\alpha_{1}-\alpha_{2}}\left(\frac{1}{\sqrt{\rho_{2}}}-\frac{1}{\sqrt{\rho_{1}}}\right)\right] \\
& \sigma_{x y}=\frac{K_{I}(\sigma)}{\sqrt{2 \pi r}} \mathfrak{R} e\left[\frac{\alpha_{1} \cdot \alpha_{2}}{\alpha_{1}-\alpha_{2}}\left(\frac{1}{\sqrt{\rho_{2}}}-\frac{1}{\sqrt{\rho_{1}}}\right)\right]+\frac{K_{I I}(\sigma)}{\sqrt{2 \pi r}} \mathfrak{R e}\left[\frac{1}{\alpha_{1}-\alpha_{2}}\left(\frac{\alpha_{1}}{\sqrt{\rho_{2}}}-\frac{\alpha_{2}}{\sqrt{\rho_{1}}}\right)\right] \\
& \rho_{j}=\cos (\theta)+i \alpha_{j} \sin (\theta), \quad j=\{1 ; 2\}
\end{aligned}
$$

Equation (16) proves that the singular field of stresses is dependent on the characteristic of materials in contrary to the isotropic case. Also, the stress intensity factor is related to the field of displacement by:

$$
\left\{\begin{array}{l}
u_{x}=2 K_{I}(\sigma) \sqrt{\frac{r}{2 \pi}} \cdot \Re e\left[\frac{1}{\alpha_{1}-\alpha_{2}}\left(\alpha_{1} p_{2} \sqrt{\rho_{2}}-\alpha_{2} p_{1} \sqrt{\rho_{1}}\right)\right]+2 K_{I I}(\sigma) \sqrt{\frac{r}{2 \pi}} \cdot \Re e\left[\frac{1}{\alpha_{1}-\alpha_{2}}\left(p_{2} \sqrt{\rho_{2}}-p_{1} \sqrt{\rho_{1}}\right)\right] \\
u_{y}=2 K_{I}(\sigma) \sqrt{\frac{r}{2 \pi}} \cdot \Re e\left[\frac{1}{\alpha_{1}-\alpha_{2}}\left(\alpha_{1} q_{2} \sqrt{\rho_{2}}-\alpha_{2} q_{1} \sqrt{\rho_{1}}\right)\right]+2 K_{I I}(\sigma) \sqrt{\frac{r}{2 \pi}} \cdot \Re e\left[\frac{1}{\alpha_{1}-\alpha_{2}}\left(q_{2} \sqrt{\rho_{2}}-q_{1} \sqrt{\rho_{1}}\right)\right]
\end{array}\right.
$$

$p_{j}$ and $q_{j}$ are defined as stated in (11).

From [13], the crack opening intensity factor and the stress intensity factor are related. Their expressions are given by the relation (18):

$$
K_{I}(\varepsilon)=C_{I} \cdot K_{I}(\sigma) \text { and } K_{I I}(\varepsilon)=C_{I I} \cdot K_{I I}(\sigma)
$$


Where $C_{I}$ and $C_{I I}(19)$ are the reduced elastic compliances for orthotropic behavior develop by [13] and cited by [16] and [17], which derive from the crack opening displacement vectors $u_{x}$ and $u_{y}$ for each mode of failure considered (Mode I and Mode II).

$$
C_{I}=4 . \Re e\left[i \frac{q_{2} \alpha_{1}-q_{1} \alpha_{2}}{\alpha_{1}-\alpha_{2}}\right] \text { and } C_{I I}=4 . \Re e\left[i \frac{p_{2}-p_{1}}{\alpha_{1}-\alpha_{2}}\right]
$$

\section{MTHETA-METHOD IN PROPAGATION}

$J$-integral [14], [15], is one of the widely applied in rate-independent quasi-static fracture analysis, as a parameter characterizing crack tip field. It could be used for linear-elastic as well as elastic-plastic material behavior and the definition is given by (20):

$$
J=G=\int_{\Gamma}\left[\left(W-\sigma_{x} \frac{\partial u}{\partial x}-\tau_{x y} \frac{\partial v}{\partial x}\right) d y+\left(\tau_{y x} \frac{\partial u}{\partial x}+\sigma_{y} \frac{\partial v}{\partial x}\right) d x\right]
$$

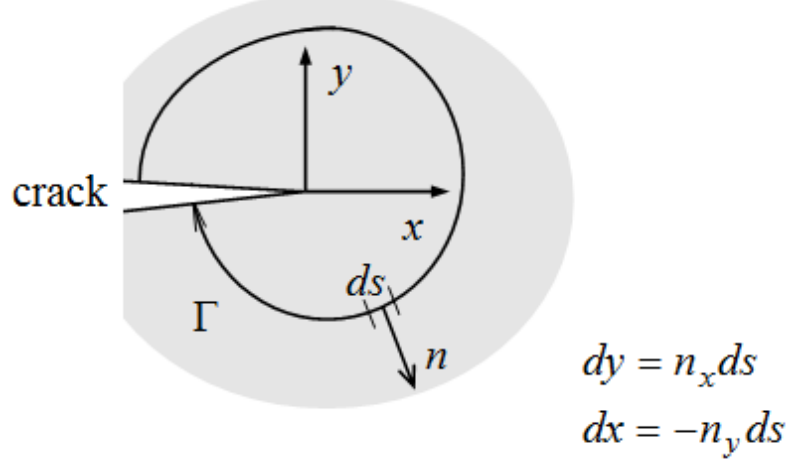

Fig. 3. Contour of integration.

$\boldsymbol{\Gamma}$ is an arbitrary curvilinear contour oriented by its normal vector $\boldsymbol{n}$ surrounding the crack type in the external failure zone, $\boldsymbol{W}$ is the strain energy density, $\sigma_{i j}$ is the component stress and $\boldsymbol{u}_{i}$ is the displacement vector. The integral form supposes that the crack is oriented in the $\boldsymbol{x}$ direction Fig. 3. However, energetic criterions necessitate a mixed-mode separation in order to isolate open and shear cinematic effects. In this regard, the generalization of the M-integral to orthotropic material has been developed by [10]. M-integral takes the following notation: 


$$
M=\frac{1}{2} \int_{C}\left[\left(\sigma_{i j, 1}^{v} \cdot u_{i}-\sigma_{i j}^{u} \cdot v_{i, 1}\right) n_{j} d C\right]
$$

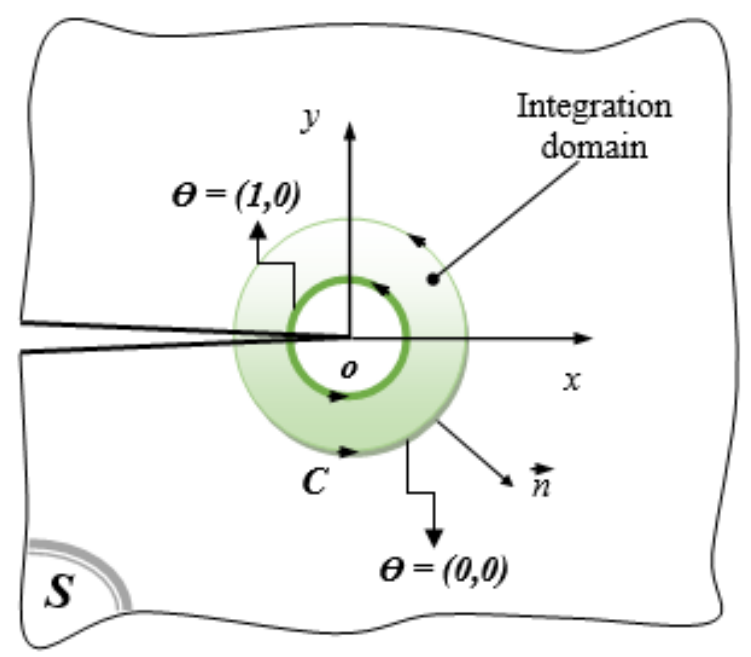

Fig. 4. Domain of integration.

Finite element analysis (FEA) is employed to transform this form in terms of a surface integral by introducing a $\theta$ scalar field which is continuous and differentiable for $\theta=1$ and $\theta=$

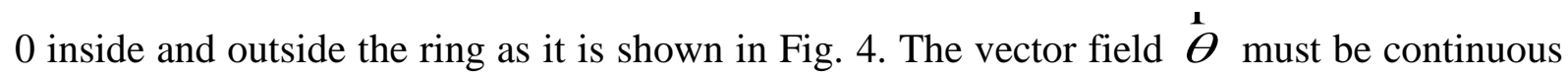
and differentiable on the considered $S$ domain. Then, the general modeling form of MO integral is given by [16], [17].

$$
M \theta=\frac{1}{2} \int_{S}\left[\left(\sigma_{i j}^{u} \cdot v_{i, k}-\sigma_{i j, k}^{v} \cdot u_{i}\right) \theta_{k, j} d S\right]
$$

where the virtual field $v$ is given by the Sih's singular form presented in equation (17). According to Dubois cited by [16], [17], the superposition principle enables to write:

$$
M \theta=C_{I} \cdot \frac{K_{I} \cdot K_{I}}{8}+C_{I I} \cdot \frac{K_{I I} \cdot K_{I I}}{8}
$$

where $C_{I}$ and $C_{I I}$ are the reduced elastic compliances in mode $I$ and mode II defining in equation (19), $K_{I}$ and $K_{I I}$ are respectively the real stress intensity factors for opening mode and shear mode. Replacing virtual and real fields, the M $\theta$-integral is assimilated to the energy release rate as follow: 
$M \theta=G=C_{I} \cdot \frac{K_{I}^{2}}{8}+C_{I I} \cdot \frac{K^{2}{ }_{I I}}{8} \Rightarrow G=G_{I}+G_{I I}$

$\mathrm{K}_{\mathrm{I}}$ and $\mathrm{K}_{\mathrm{II}}$ can be obtained independently, let perform two distinct calculations from the MӨintegral:

$$
K_{I}=\frac{8 \cdot M \theta\left(K_{I}=1 ; K_{I I}=0\right)}{C_{I}} \text { and } K_{I I}=\frac{8 \cdot M \theta\left(K_{I}=0 ; K_{I I}=1\right)}{C_{I I}}
$$

Expression (25) inside (24), compute the energy release rate part for each mode.

\section{GEOMETRY OF SENB SIMULATION SPECIMEN}

The geometry of the specimen is firstly generated according to the SENB specimen. This specimen is adapted to model easily in three point bending the evolution of crack. The obtained specimen dimensions are presented in Fig. 5 with the following values base on [18]

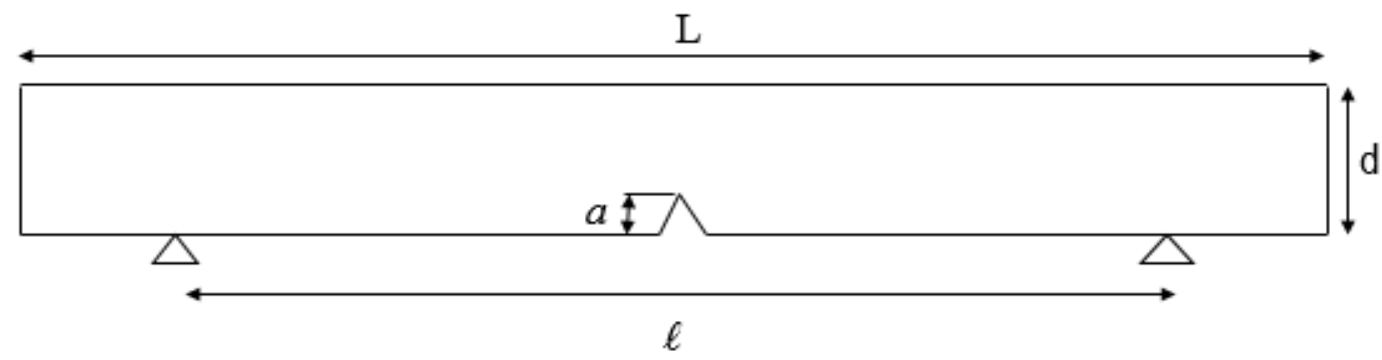

work.

Fig. 5. Dimension of specimen.

It's used to evaluate crack displacement on the hardwoods Lovoa trichilioides and Triplochiton scleroxylon under loading force control. The specimen is used in computational finite elements method to generate the mode I and II fracture toughness in the RL planes. The 2-D finite element mesh of the specimen obtained for deformation and displacement respectively in pure mode I and II simulation are shown in Fig. 6. 


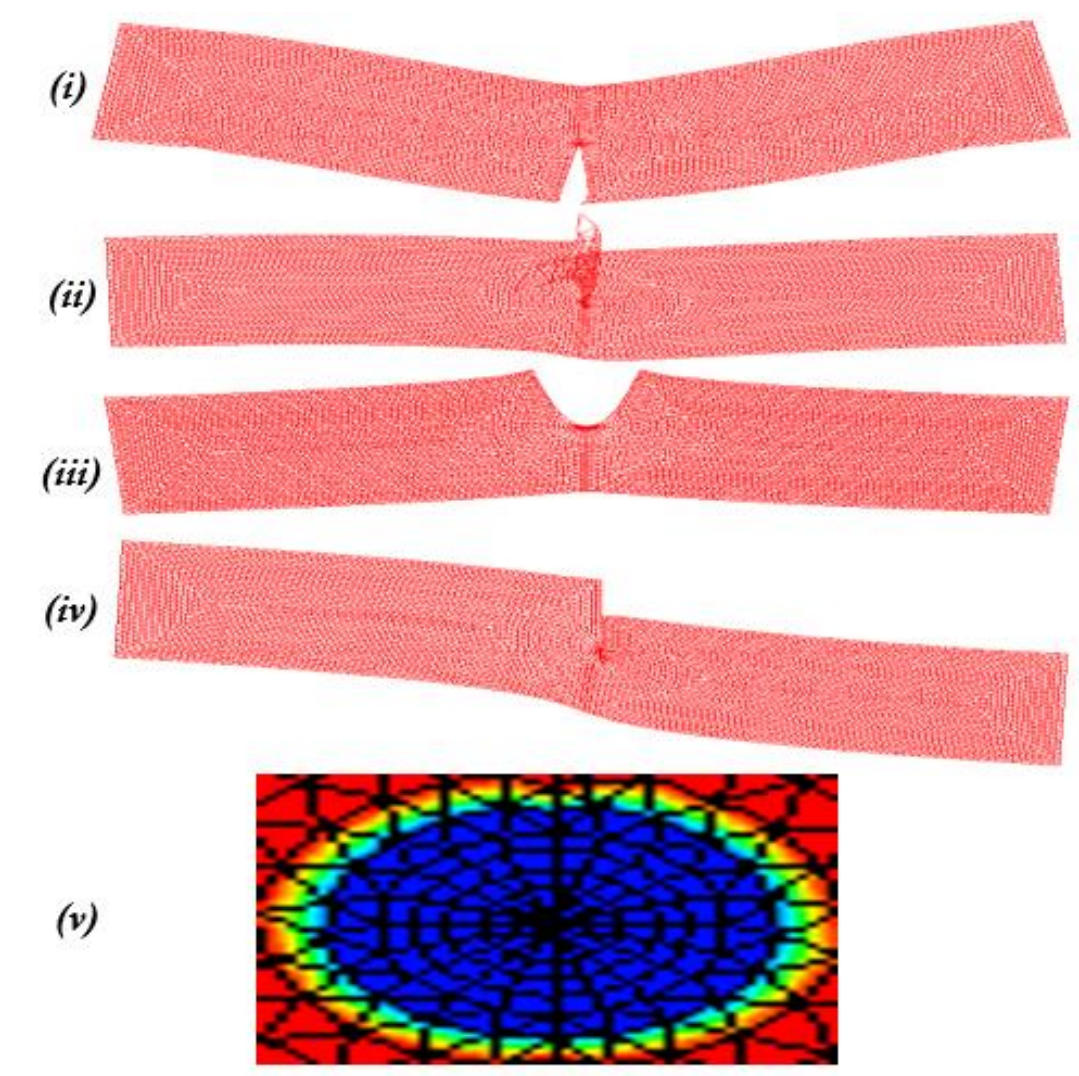

Fig. 6. Finite element mesh of specimen: (i) Mode I deformation shape, (ii) Mode II deformation shape, (iii) Open mode virtual displacement, (iv) Shear mode virtual displacement, $(v)$ Circular mesh around crack tip.

The geometry is calculated using a plane stress assumption, using a unitary loading force corresponding to the determination of stress intensity factor and the energy release rate values. This finite element mesh in which a circular discretization around the crack tip (Fig. 6 $(v)$ ), allows to easily define the $\theta$ vector field and the integration crown using parametric elements. M $\theta$-integral performed the virtual finite element displacement fields (Fig. 6 (iii) and (iv)) and deformation (Fig. 6 (i) and (ii)) for open and shear mode respectively. The computation is limited for an elastic response according to 2-D formulation and the characteristics of the hardwoods are taken as follow:

$$
E_{\mathrm{L}}=7260 \mathrm{MPa}, \mathrm{E}_{\mathrm{R}}=893 \mathrm{MPa}, \mathrm{G}_{\mathrm{LR}}=552 \mathrm{MPa}, v_{\mathrm{RL}}=0.4, \text { Density }=380 \mathrm{Kg} / \mathrm{m}^{3}
$$

Equation (27) is the characteristic of Triplochiton scleroxylon hardwoods were $E$ is the Young module, $G$ and $v$ are shear module and Poisson coefficient respectively. 
$E_{\mathrm{L}}=10460 \mathrm{MPa}, \mathrm{E}_{\mathrm{R}}=1287 \mathrm{MPa}, \mathrm{G}_{\mathrm{LR}}=795 \mathrm{MPa}, v_{\mathrm{RL}}=0.4$, Density $=530 \mathrm{Kg} / \mathrm{m}^{3}$

The hardwoods Lovoa trichilioides properties are cited in (28) with the same components define previously.

\section{RESULTS AND DISCUSSIONS}

The simplified numerical routine (flow diagram) used to compute the fracture parameters based on $M \theta$-integral is sketched in Fig. 7. The Fig. 8 below evaluates the path independence of the methods on the specimen according to crown. By considering different crack orientations, it is observed the unvarying results versus different crowns for energy release rate evolution. Across singularity in the crack tip or near zero the mechanical fields cause a disturbance through the integration process for the crown 0 , which is the origin of the

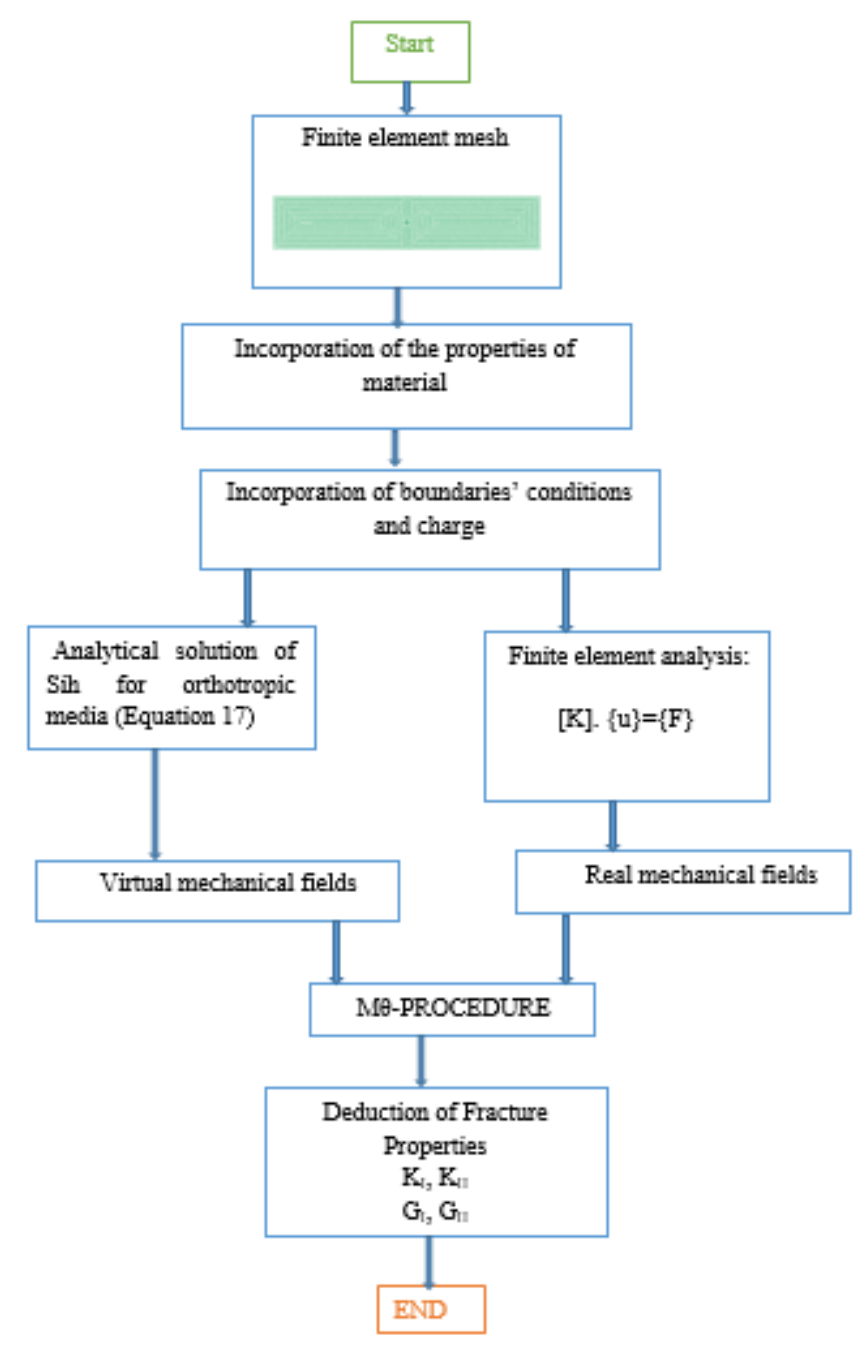

perturbation. Nevertheless, there is a great stability with the increase of crown sizes.

Fig. 7. Flow diagram on computing fracture parameters. 
This satisfied the theoretical assumption of path independence in agreement with [19] work. From the result, in what follows crown six, will be used to performed calculations of the stress intensity factors and energy release rate.

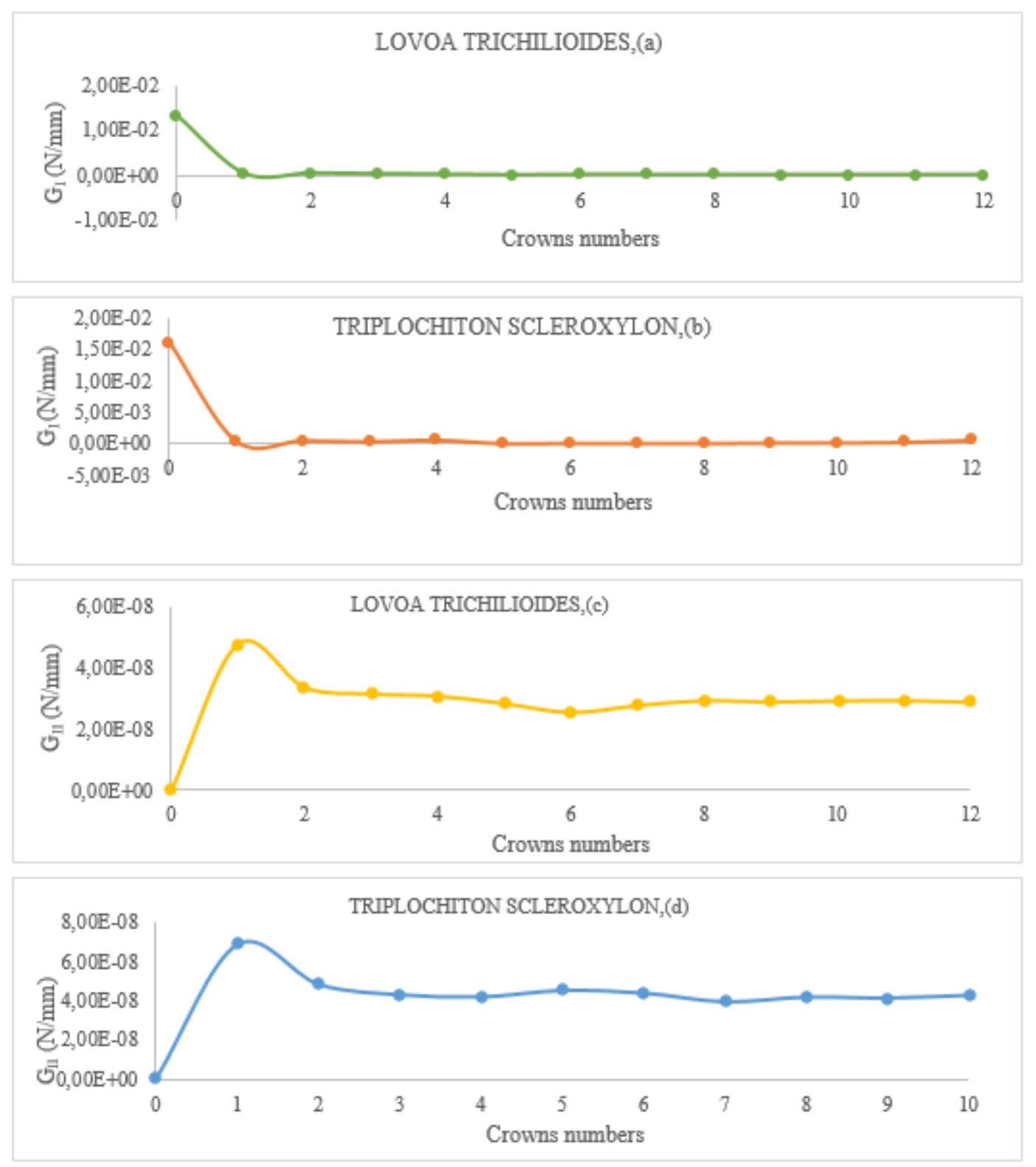

Fig. 8. Path independence on triplochiton scleroxylon and lovoa trichilioides: (a), (b) Mode I and (c), (d) Mode II.

From Fig. 9 (i), (ii) and Fig. 10 (a), (b) general observation indicated the decreasing of the energy release and stress intensity factor with the growing of the crack. This behavior was obtained by [20], [21]. Hence the total energy of the two wood is reduced to the released strain energy consequences of the decrement and induced their brittle fracture. 

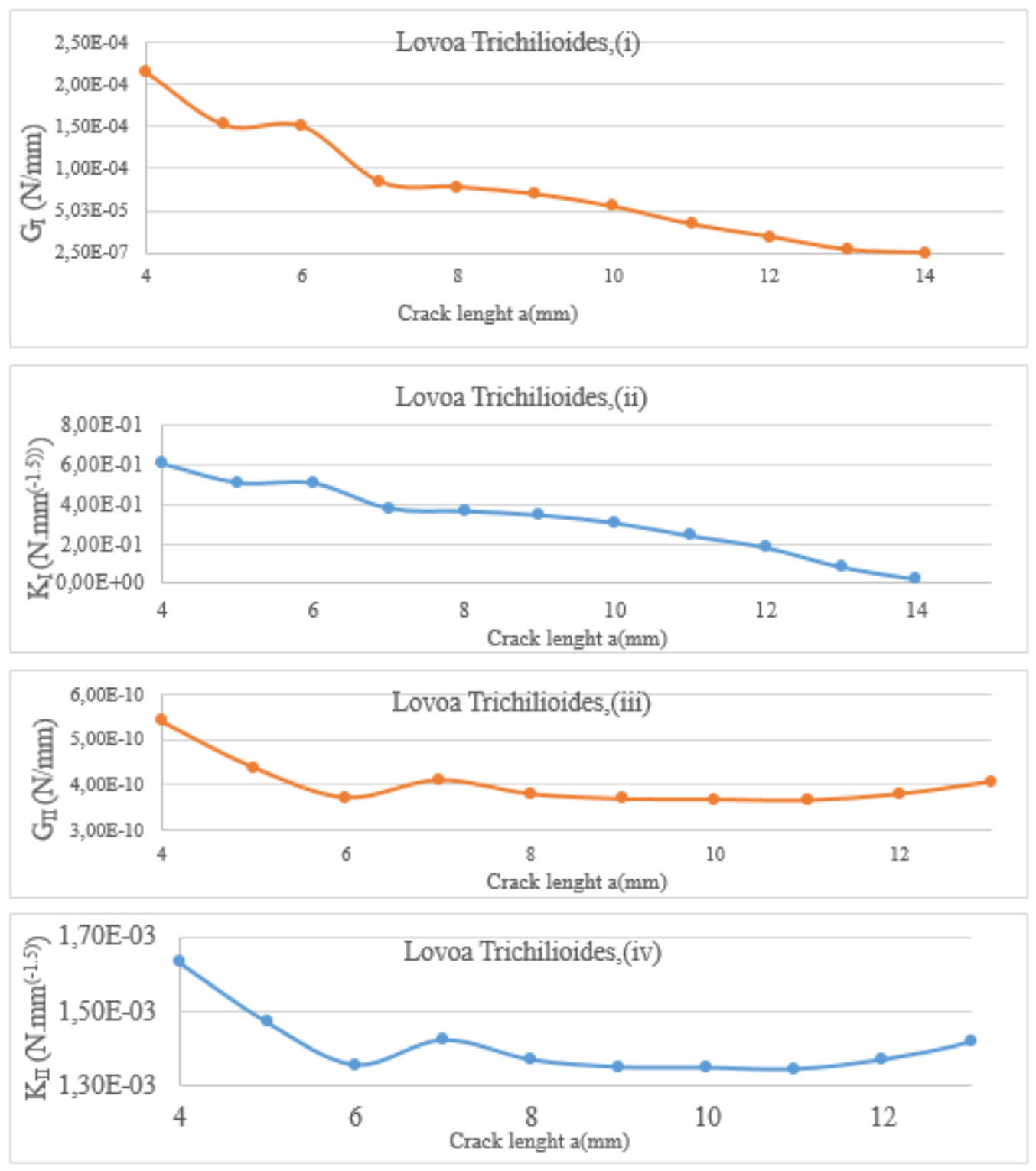

Fig. 9. (i), (iii) Energy release rate and (ii), (iv) Stress intensity factor versus crack growth position for lovoa trichilioides in mode I and II.

This type of result would occur in a "fixed-grips" apparatus with the applied load and the apparatus clamped into position. The same arguments can be exactly being applied for a "dead-weight" loading, where the fracture surface energy corresponds to a decrease in potential energy of the loading system [21]. 

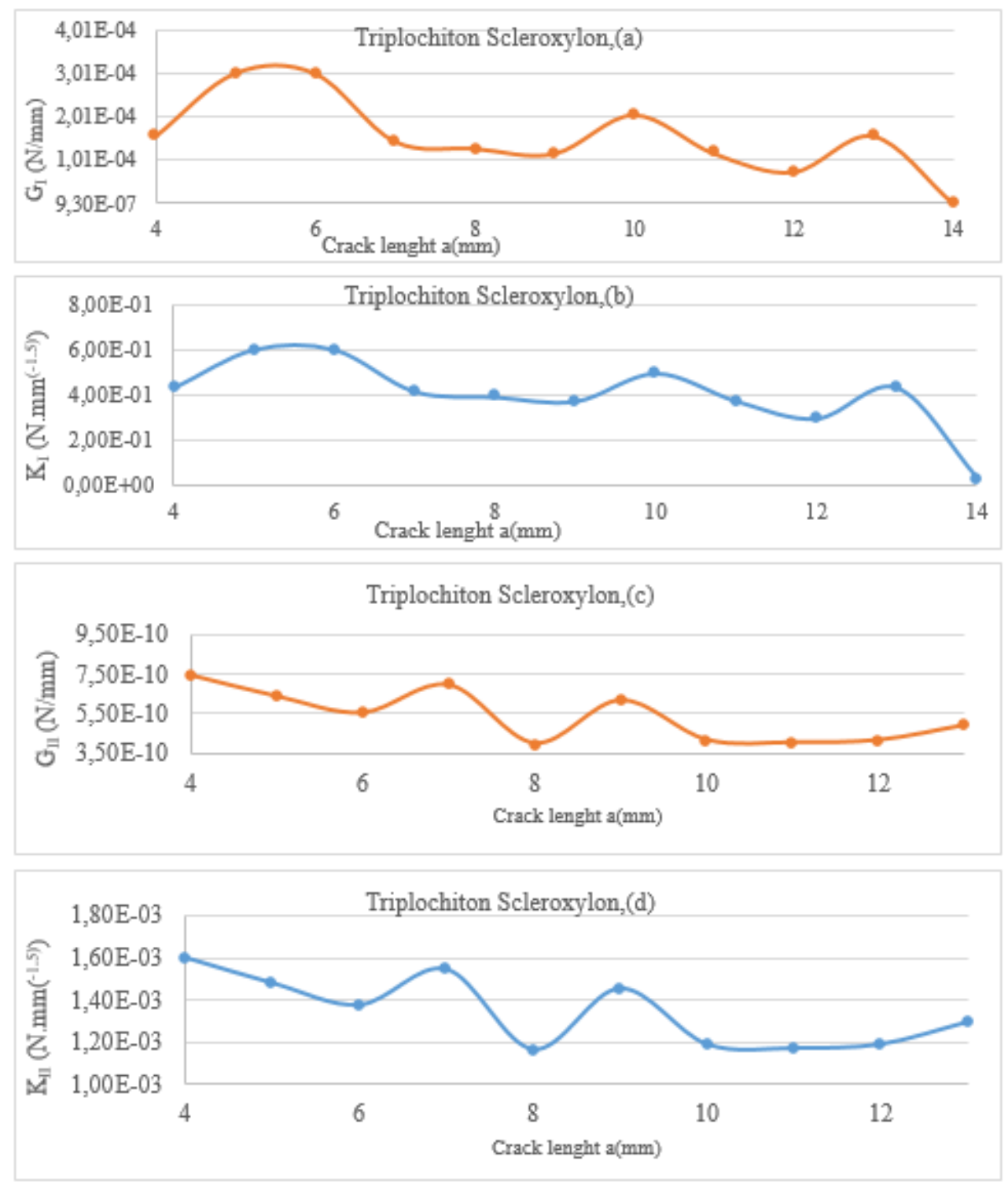

Fig. 10. (a), (c) Energy release rate and (b), (d) Stress intensity factor versus crack growth position for triplochiton scleroxylon in mode I and II.

The apparent drop in mode II toughness of stress intensity factor and energy release rate (Fig. 9 (iii), (iv)) and (Fig. 10 (c), (d)) observed as crack length increases for the two hardwoods were obtained by [8] on four-point bending end notched flexure (4ENF) tests. The causes of this behavior is not yet known but, [8] observed that the crack tends to open under large displacements and tended to migrate away from the mid-thickness of the specimen and towards face of tension. They remark that mode I fracture in addition induce to the intended mode II fracture for the opening of the crack and the measured of fracture toughness is modify by the mixed mode. In this respect, this study reveals the decreasing of the energies 
for the both two modes of fracture; which imply future investigation of the effect of mixed mode to overcome some conclusion on these behaviors.

\section{CONCLUSION AND PERSPECTIVE}

In this study, the evolution of energy release rate and stress intensity factors of two woods species have been investigated on bending. The finite element mesh of the two hardwoods in different modes were presented. The independently computation results of the stress intensity factor and the energy release rate for mode I and mode II fracture are proved. The pathindependence of $\mathrm{M} \theta$-integral result have been showed. It has been observed the quasi decrement of energy release rate and stress intensity factors of the both two hardwoods species. The future investigation will be consecrated to study mixes mode fracture on the two hardwoods to evaluate his effect and finally it will be important to make an experimental setup result to compare with the numerical results.

\section{REFERENCES}

[1] P.K. Talla, E. Foadieng, W.C.M. Fouotsa, M. Fogue, S. Bishweka, K.E. Ngarguededjim, F.S. Alabeweh, "Contribution to the study of Entandrophragma cylindricum sprague and Lovoa Trichilioiides harms long term behavior", Revue Scientifique et Technique Forêt et Environnement du Bassin du Congo, vol. 5, pp. 10-25, 2015.

[2] H. Kouefouet, G. Bawe, W.C.M. Fouotsa, P.K. Talla, F. J. A. Mukam, E. Foadieng, "Probabilistic approach of the failure of lovoa trichilioides and triplochiton scleroxylon". Wood Research, vol. 63, no. 2, pp. 273-286, Jan. 2018.

[3] H. Danielsson, "Perpendicular to grain fracture analysis of wooden structural elements models and applications”, Doctoral Thesis, Dept. Const. Sci., Lund Univ, SM, 2013.

[4] F. Eyma, P-J. Méausoone, P. Martin, "Study of the properties of thirteen tropical wood species to improve the prediction of cutting forces in mode B", Ann. For. Sci., vol. 61, pp. 55-64, 2004.

[5] S. Aicher, "Process zone length and fracture energy of spruce wood in Mode-I from size effect", Wood and Fiber Science, vol. 42, no. 2, pp. 237-247, April 2010.

[6] E.S. Alami, E.M. Mouridi, T. Laurent, G. Calchera, A. Famiri, A. Hakam, B. Kabouchi, J. Gril, "Fracture energy of wood and root burl wood of thuya (Tetraclinis articulata)". Journal of Tropical Forest Science, vol. 25, no. 2, pp. 166-174, 2013. 
[7] B. Odounga, R. Moutou Pitti, E. Toussaint, M. Grédiac, "Mode I fracture of tropical woods using grid method", Theoretical and Applied Fracture Mechanics, vol. 95, pp. 117, Feb. 2018.

[8] R.J. Brambleby, L.A. Louca, S.E. Mouring, "Influence of loading rate on the mode II fracture toughness of vinyl ester GRP”, Composites: Part A, vol. 93, pp. 153-162, 2017.

[9] R. Moutou Pitti, F. Dubois, N. Sauvat, C. Petit, «Propagation de fissure en mode mixte dans un matériau viscoélastique orthotrope : Approche numérique et analytique par

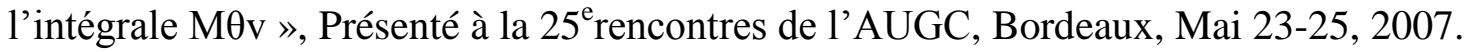

[10] R. Moutou Pitti, F. Dubois, C. Petit, N. Sauvat, O. Pop, “A new M-integral parameter for mixed-mode crack growth in orthotropic viscoelastic material”, Engineering Fracture Mechanics, vol. 75, pp. 4450-4465, 2008.

[11] R. Moutou Pitti, C. Chazal, F. Labesse-Jied, Y. Lapusta, C. E. Ventura, W. C. Crone, C. Furlong, "Stress Intensity Factors for Viscoelastic Axisymmetric Problems Applied to Wood", Experimental and Applied Mechanics, vol. 4, pp. 89-96, 2012.

[12] H. Boulazreg, « Modélisation de la fissuration fragile par une formulation mixte hybride ; évaluation des coefficients de concentration de contraintes et du taux de restitution d'énergie », Thèse de Doctorat, École Centrale de Lyon, GC, 1991.

[13] F. Dubois, «Modélisation du comportement mécanique des milieux viscoélastiques fissurés : application au matériau bois », Thèse de Doctorat, Dept. Gen. Civ., Limoges Univ, 1997.

[14] J. R. Rice, "A path independent integral and the approximate analysis of strain concentration by notches and cracks", REPORT E39, Div. Eng., Brown Univ and ARPA, May 1967.

[15] J. R. Rice, "A path independent integral and the approximate analysis of strain concentration by notches and cracks", Journal of Applied Mechanics, vol. 35, pp. 379386, June 1968.

[16] R. Moutou Pitti, F. Dubois, C. Petit, N. Sauvat, "Mixed mode fracture separation in viscoelastic orthotropic media: numerical and analytical approach by the M $\theta$ v-integral", Int J Fract, vol. 145, pp. 181-193, 2007.

[17] M. Meite, «Caractérisation des paramètres de fissuration par un couplage corrélation d'images et éléments finis », Thèse de Doctorat, Dept. Gen. Civ., Limoges Univ, 2012.

[18] M. Lahlou, A. Hachim, H. Ouaomar, N. Mouhib, E.M. Ghorba, "Procedure for the numerical modeling of the specimen (SENB) using CAST3M calculation code", 
International Journal of Innovation and Scientific Research, vol. 19, no. 2, pp. 259-266, 2015.

[19] A. A. El Malki, «Influence du chargement sur la propagation en fatigue de fissures courtes dans un acier de construction navale », Thèse de Doctorat, Dept. Sci. Ing., Metz Univ, MM, 2005.

[20] M. Benguediab, B.B. Bouiadjra, M. Elmeguenni, «Modélisation par éléments finis du comportement d'un polymère semi cristallin : Polyéthylène à haute densité », Présenté au $9^{\text {ième }}$ Congrès de Mécanique, Marrakech-Maroc, Avril 21-24, 2009.

[21] A.C. Fischer-Cripps, Introduction to contact mechanics, Hardcover, XXII: Springer, 2007, ch. 2, pp. 1-19.

\section{Author's formal address:}

1- Mr. KOUEFOUET Hervice ( $\mathrm{PhD}$ student); Faculty of Science, UR-2MSP, University of Dschang; Cameroon; kouefoueth@yahoo.fr; hervice.kouefouet@univdschang.org;

2- Dr. MABEKOU Takam Jeanne Sandrine (Lecturer), Faculty of Science, UR-2MSP, University of Dschang; Cameroon; sandrinengamga@yahoo.com;

3- Pr. MUKAM Fotsing Joseph Albert (Associate professor); Ecole Normale Supérieure (ENS), University of Yaoundé I, Cameroon; mukam_fotsing_j_a@yahoo.com

4- Pr. MOUTOU Pitti Rostand (Associate professor) ; Université Clermont Auvergne, CNRS, SIGMA Institut Pascal, 63000 Clermont-Ferrand, France ; rostand.moutou_pitti@uca.fr; rostand.moutou.pitti@gmail.com

5- Pr TALLA Pierre Kisito (Professor); Faculty of Science, UR-2MSP, University of Dschang; Cameroon; tpierrekisito@yahoo.com.

> UR-2MSP : Unité de Recherche de Mécanique et de Modélisation des systèmes physiques (Faculty of Sciences), University of Dschang, Cameroon ; Institutional webpage : Website : http://www.univ-dschang.org.

$\boldsymbol{D}$ ENS : École Normale Supérieure ; Institutional webpage: Website : http://www.uyl.uninet.cm. 